\title{
Roadmap and standard operating procedures for biobanking and discovery of neurochemical markers in ALS
}

\author{
MARKUS OTTO ${ }^{1 *}$, ROBERT BOWSER ${ }^{2 *}$, MARTIN TURNER $^{3 *}$, JAMES BERRY $^{4}$, \\ JOHANNES BRETTSCHNEIDER ${ }^{1}$, JAMES CONNOR ${ }^{5}$, JÚLIA COSTA ${ }^{6}$, \\ MERIT CUDKOWICZ ${ }^{4}$, JONATHAN GLASS ${ }^{7}$, OLAF JAHN ${ }^{8}$, STEFAN LEHNERT $^{1}$, \\ ANDREA MALASPINA $^{9}$, LUCILLA PARNETTI ${ }^{10}$, AXEL PETZOLD ${ }^{11}$, PAMELA SHAW ${ }^{12}$, \\ ALEXANDER SHERMAN ${ }^{4}$, PETRA STEINACKER ${ }^{1}$, SIGURD SÜßMUTH ${ }^{1}$, \\ CHARLOTTE TEUNISSEN ${ }^{13}$, HAYRETTIN TUMANI ${ }^{1}$, ANNA WUOLIKAINEN ${ }^{14}$, \\ ALBERT LUDOLPH' ${ }^{1}$ - AS MEMBERS OF THE 'VOLCANO GROUP'**
}

\begin{abstract}
${ }^{1}$ Department of Neurology, University of Ulm, Germany, ${ }^{2}$ Division of Neurology, Barrow Neurological Institute, St. Foseph's Hospital and Medical Center, Phoenix, Arizona, USA, ${ }^{3}$ Oxford University Nuffield Department of Clinical Neurosciences, Fohn Radcliffe Hospital, Oxford, UK, ${ }^{4}$ Department of Neurology, Massachusetts General Hospital, Harvard Medical School, Boston, Massachusetts, ${ }^{5}$ George M. Leader Family Laboratory, Department of Neurosurgery, Pennsylvania State University College of Medicine, Hershey, Pennsylvania, USA, ${ }^{6}$ Laboratory of Glycobiology, Instituto de Tecnologia Química e Biológica, Oeiras, Portugal, ${ }^{7}$ Emory University School of Medicine, Atlanta, Georgia, USA, ${ }^{8}$ Max-Planck-Institute of Experimental Medicine, Goettingen, Germany, ${ }^{9}$ Centre for Neuroscience and Trauma, Blizard Institute of Cell and Molecular Science, Queen Mary Hospital, University of London, UK, ${ }^{10}$ Centre for Memory Disturbances, Laboratory of Clinical Neurochemistry, Section of Neurology, University of Perugia, Perugia, Italy, ${ }^{11}$ UCL Institute of Neurology, Department of Neuroimmunology, The National Hospital for Neurology and Neurosurgery, Queen Square, London, UK and Department of Neurology, VU University Medical Centre, Amsterdam, The Netherlands, ${ }^{12}$ Academic Unit of Neurology, University of Sheffield Medical School, Sheffield, UK, ${ }^{13}$ Neurological Laboratory, Department of Clinical Chemistry, VU University Medical Centre, Amsterdam, The Netherlands, and ${ }^{14}$ Computational Life Science Cluster, Department of Chemistry, Umeå University, Umeå, Sweden
\end{abstract}

\begin{abstract}
Despite major advances in deciphering the neuropathological hallmarks of amyotrophic lateral sclerosis (ALS), validated neurochemical biomarkers for monitoring disease activity, earlier diagnosis, defining prognosis and unlocking key pathophysiological pathways are lacking. Although several candidate biomarkers exist, translation into clinical application is hindered by small sample numbers, especially longitudinal, for independent verification. This review considers the potential routes to the discovery of neurochemical markers in ALS, and provides a consensus statement on standard operating procedures that will facilitate multicenter collaboration, validation and ultimately clinical translation.
\end{abstract}

Key words: Biomarker, biobank, proteomics, cerebrospinal fluid, brain bank

\section{Introduction}

It has been nearly half a century since the first published putative biomarker for amyotrophic lateral sclerosis (ALS), namely the observation of altered serum levels in response to oral arginine compared with controls (1). Many and varied candidates have subsequently emerged as a result of advances in molecular biological and other analytical techniques $(2-4)$. This has resulted from a greater understanding of the underlying molecular mechanisms, but also improved clinical recognition and characterization of the heterogeneous syndrome of ALS (5). Currently riluzole is the only disease-modifying drug licensed for treatment of ALS and the focus of management

\footnotetext{
${ }^{*} \mathrm{M}$. Otto, R. Bowser and M. Turner contributed equally to this article.

**The First Workshop on Neurochemical Markers in ALS, held at Reisensburg Castle, Ulm, Germany $14-16$ July 2010.

Correspondence: M. Otto, Department of Neurology, University of Ulm, Oberer Eselsberg 34, 89081 Ulm, Germany. Fax: 4973150063012.

E-mail: markus.otto@uni-ulm.de
} 
Table I. ALS ideal biomarker characteristics.

1. Sensitive and specific for the heterogeneous syndrome of ALS

2. Detectable prior to the onset of significant wasting/ weakness

3. Discriminate between clinical phenotypes based on:

a) Upper and lower motor neuron involvement, e.g. PMA and PLS

b) Patterns of regional involvement, e.g. flail arm/leg, PBP

c) Cognitive involvement, i.e. ALS versus ALS-FTD

d) Extremes of survival, i.e. 'aggressive' versus 'benign' course

4. Able to predict regional involvement and the pattern of spread in advance:

a) Bulbar dysfunction for early gastrostomy

b) Respiratory dysfunction for early NIV (possibly diaphragm pacing)

c) Cognitive impairment

5 Change in a predictable way with disease progression

6. Sensitivity to confidently judge therapeutic response within weeks of challenge

7. Easily accessible and affordable technology

8. Practical to measure in the physically disabled patient

ALS: amyotrophic lateral sclerosis; FTD: frontotemporal dementia: NIV: non-invasive ventilation; PBP: progressive bulbar palsy; PMA: progressive muscular atrophy; PLS: primary lateral sclerosis.

from diagnosis is palliative. Future therapeutic agents may come with inherent toxicities that require monitoring and adjustment of dose or choice of drug. As well as radically changing the nature and focus of ALS clinics, such a therapeutic era will place even greater reliance on robust biomarkers upon which to base such decisions. The concept of a recognizable 'ALS signature' is attractive, with the corollary of earlier, perhaps ultimately preventative, intervention. However, the inherent clinical heterogeneity, the lack of understanding of those at risk and where the 'clinical horizon' lies in relation to the earliest pathogenic events, present major challenges to translation of candidates into clinically meaningful biomarkers.

\section{What is the aim?}

The ideal ALS biomarker characteristics are summarized in Table I. Biomarkers with diagnostic utility may assist primary care physicians or general neurologists to more rapidly refer a patient to a specialized ALS clinic. Those sensitive to disease activity might shorten clinical trials, more rapidly halting non-effective drug trials and potentially enabling patients to enrol in multiple trials. ALS biomarkers have potential value on several levels, but the clinical benchmarks are already high (see Table II). Moreover, the history of biomarker discovery in human disease to date is sobering, with $<0.01 \%$ of the number of published papers containing multiple candidates estimated to have been translated into biomarkers in routine clinical use (6).

National and international collaborations are required to obtain the numbers of samples necessary

Table II. Categories of biomarker in ALS.

\begin{tabular}{|c|c|c|c|}
\hline Category & Purpose & Current benchmark & Challenges \\
\hline Diagnostic & $\begin{array}{l}\text { To reduce diagnostic delay } \\
\text { (current mean } \sim 1 \text { year from } \\
\text { symptom onset); } \\
\text { To more rapidly refer patients to } \\
\text { neuromuscular specialist; } \\
\text { To distinguish ALS from mimic } \\
\quad \text { disorders; } \\
\text { Permit earlier therapeutic } \\
\text { intervention }\end{array}$ & $\begin{array}{l}\text { Clinical acumen; } \\
\text { ALS neurologists in tertiary } \\
\text { referral centers } \\
\text { probably }>97 \% \text { sensitive and } \\
\text { specific, but predominantly as } \\
\text { a result of referral delay }\end{array}$ & $\begin{array}{l}\text { Who are the 'at risk' population? } \\
\text { Where is the 'clinical horizon' in } \\
\text { relation to pathogenesis? } \\
\text { The true mimics are not healthy } \\
\text { controls, but pure LMN and } \\
\text { pure UMN syndromes }\end{array}$ \\
\hline Prognostic & $\begin{array}{l}\text { To identify those with poor } \\
\text { prognosis who may require } \\
\text { earlier intervention or care } \\
\text { planning. } \\
\text { Identify the minority for whom } \\
\text { cognitive involvement likely to } \\
\text { be significant and intrusive }\end{array}$ & $\begin{array}{l}\text { Prognostic modeling of survival } \\
\text { with clinical parameters }\end{array}$ & $\begin{array}{l}\text { Prognostic modeling based on } \\
\text { tertiary referral center data; } \\
\text { Need to establish how earlier } \\
\text { intervention in 'aggressive } \\
\text { ALS' might actually be } \\
\text { beneficial. } \\
\text { Those with ALS-FTD often } \\
\text { present with early dementia } \\
\text { before motor features are } \\
\text { apparent }\end{array}$ \\
\hline Monitoring & $\begin{array}{l}\text { To provide a marker of early } \\
\text { therapeutic benefit in clinical } \\
\text { trials. } \\
\text { Reduce size and duration of } \\
\text { studies. } \\
\text { Reduce need for placebo arms }\end{array}$ & $\begin{array}{l}\text { Survival as main endpoint, } \\
\text { which requires trials of }>200 \\
\text { patients over } 12-24 \text { months. } \\
\text { ALSFRS-R and FVC are } \\
\text { alternative surrogate } \\
\text { endpoints }\end{array}$ & $\begin{array}{l}\text { Heterogeneous syndrome of ALS } \\
\text { may distort all trial outcome } \\
\text { measures due to different } \\
\text { efficacy across phenotypes }\end{array}$ \\
\hline Mechanistic & $\begin{array}{l}\text { Biomarkers may provide clues to } \\
\text { pathogenesis via pathway } \\
\text { analysis and thence provide } \\
\text { new candidates }\end{array}$ & $\begin{array}{l}\text { Cellular inclusions, e.g. TDP-43 } \\
\text { and FUS indicate abnormal } \\
\text { RNA processing; may be } \\
\text { important in pathogenesis }\end{array}$ & $\begin{array}{l}\text { Distinguishing pathogenic } \\
\text { pathways from epi-phenomena }\end{array}$ \\
\hline
\end{tabular}

ALS: amyotrophic lateral sclerosis; ALSFRS-R: revised ALS functional rating score;

FTD: frontotemporal dementia; FVC: forced vital capacity. LMN: lower motor neuron; UMN: upper motor neuron 
to perform large biomarker validation studies for rare disorders like ALS (2). A recent initiative resulted in consensus guidelines for neuroimaging biomarkers in ALS (7), and ALS biobanking efforts have been initiated in some countries, e.g. the Northeast ALS consortium (NEALS) BioBank (www.alsconsortium.org) (8). However, standardized protocols of sample collection and annotated clinical information are not currently uniform and limit the ability to share samples and information. The Alzheimer's Disease Neuroimaging Initiative (ADNI) standardized procedures for the collection, processing, storage and shipment of biofluid samples, from which a consortium identified CSF biomarkers for the early disease currently being validated in large studies. In addition, a consensus protocol to collect and bank CSF for chronic inflammatory diseases and for a wider diagnostic spectrum of disorders has been recently published (9).

\section{Study design}

The design of ALS biomarker studies is critical, whether the primary outcome is the generation of candidate biomarkers, or establishing the place of pre-defined markers within the current clinical management or therapeutic intervention (Figure 1).

\section{Cross-sectional studies and homogeneity}

Comparison of patients with a selected control group at a single time-point may identify biomarkers that are enriched or depleted in ALS. This strategy can be useful for identifying potential diagnostic markers or pathogenic pathways. These designs will not have value for identifying markers of therapeutic response or prognosis without continued clinical monitoring. The yield from group comparison may be reduced as a consequence of the heterogeneity in ALS (and compounded by small sample size); however, attempts to select patients with homogeneous phenotype are not without their own problems. Grouping of patients by site of symptom onset (e.g. bulbar versus limb) may have value as a broad prognostic marker. Realistically, however, there is no evidence for a distinct pathogenesis in terms of site of onset, nor between regional phenotypes, e.g. 'flail arm', so separation of groups by onset phenotype may be artificial. Equally, rigid adherence to revised El Escorial criteria categories does not equate to homogeneity in terms of prognosis. Furthermore, such criteria exclude those without mixed upper and lower motor neuron signs clinically, despite general acceptance that Progressive Muscular Atrophy and Primary Lateral Sclerosis form a continuum with ALS at a pathological level. Age at symptom onset and other established prognostic surrogates such as referral delay, are potential confounds within an otherwise homogeneous ALS group. While the exploration of subgroups stratified according to a variety of criteria
Steps of biomarker discovery / implementation

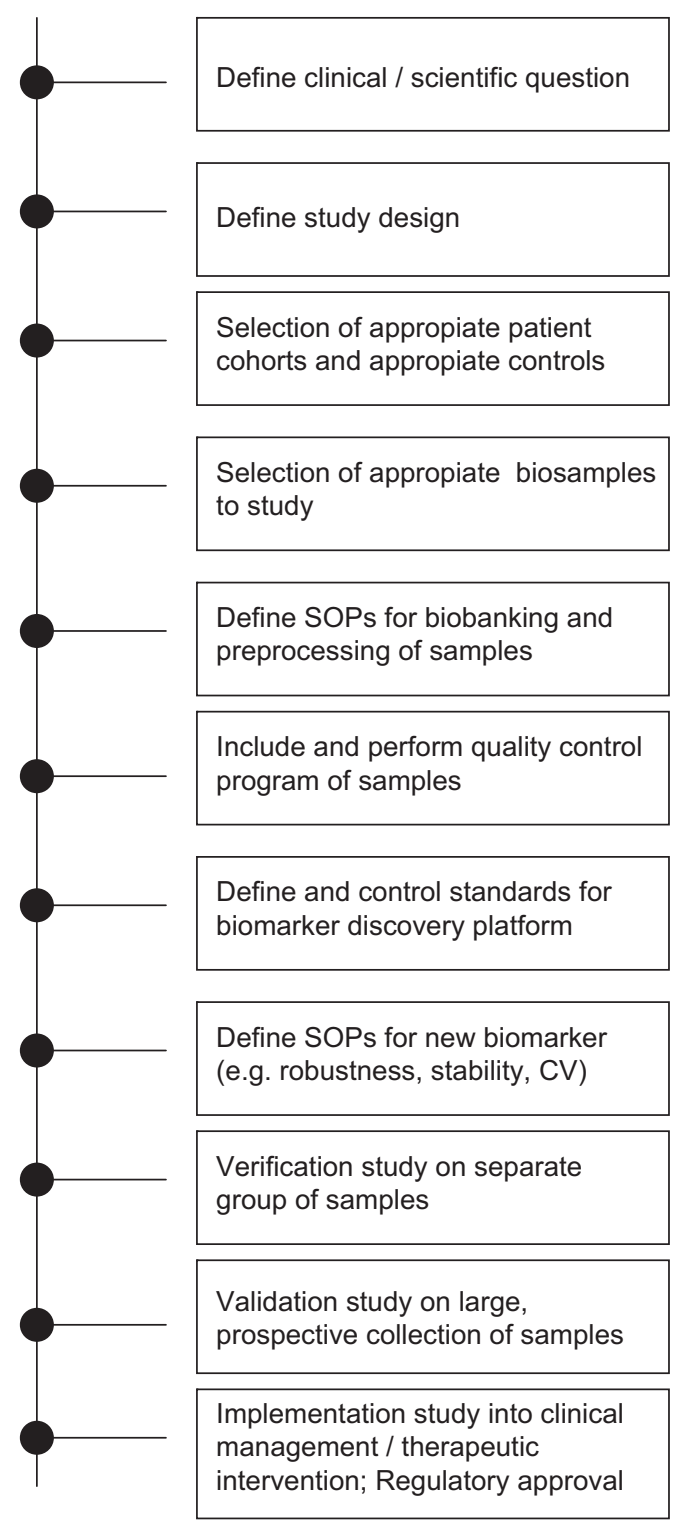

Figure 1. Roadmap for biomarker discovery, biobanking and implementation of neurochemical markers in ALS.

may still be of interest, a 'real world' approach to biomarker development is advocated that seeks to be meaningful in translation to clinical practice, reflecting the natural spectrum of clinical phenotype common to many diseases within diverse human populations. This approach may require multivariate modeling in larger groups of patients to control for known prognostic factors, and emphasizes the need for rigorous (though not unduly onerous) clinical information gathering.

\section{Choice of controls}

The selection of controls must be based on the scientific question, and not simply on the ease of availability of samples. Possible sources are listed in Table III. Control samples as a rule are likely to be more meaningful if matched for age and gender. 
Table III. Types of control sample in ALS biomarker studies.

\begin{tabular}{|c|c|c|}
\hline Category of control & Advantage & Disadvantage \\
\hline Healthy & $\begin{array}{l}\text { 'Pure' sample; } \\
\text { greater sensitivity; } \\
\text { spouses of patients easily } \\
\quad \text { available resource }\end{array}$ & $\begin{array}{l}\text { Ethical approval for invasive procedures } \\
\text { especially spinal tap, although research } \\
\text { experience is reassuring and should be } \\
\text { proactively emphasized in IRB/REC } \\
\text { applications. } \\
\text { Age-matching additionally challenging. } \\
\text { Possibly reduced specificity }\end{array}$ \\
\hline $\begin{array}{l}\text { 'Ward traffic' i.e. as part of routine } \\
\text { investigation with additional } \\
\text { sampling volume }\end{array}$ & $\begin{array}{l}\text { Availability. } \\
\text { Ethical approval and consent } \\
\text { easier to obtain as it is part } \\
\text { of routine care }\end{array}$ & $\begin{array}{l}\text { Diagnosis may be idiopathic e.g. 'headache' } \\
\text { or 'parasthesiae', with a need for } \\
\text { follow-up to categorize later as further } \\
\text { information comes to light (and ethical } \\
\text { approval in this respect). } \\
\text { Experience from lumbar drainage in } \\
\text { Normal Pressure Hydrocephalus suggests } \\
\text { multiple confounds may exist }\end{array}$ \\
\hline Other neurodegenerative disorders & $\begin{array}{l}\text { Explore specificity of putative } \\
\text { markers, especially neuronal. } \\
\text { FTD particularly relevant to } \\
\text { ALS }\end{array}$ & $\begin{array}{l}\text { Scarcity. } \\
\text { Relevance to ALS? } \\
\text { Poor clinical diagnostic accuracy }\end{array}$ \\
\hline $\begin{array}{l}\text { Clinical 'mimics' e.g. multifocal } \\
\text { motor neuropathy with conduction } \\
\text { block (MFMN), Kennedy's } \\
\text { syndrome, cervical spondylosis }\end{array}$ & $\begin{array}{l}\text { Value as source for truly } \\
\text { diagnostic biomarkers }\end{array}$ & $\begin{array}{l}\text { Scarcity. } \\
\text { Limited value in current clinical practice. } \\
\text { Mimics may not be neurodegenerative in } \\
\text { pathogenesis e.g. MFMN, spondylosis }\end{array}$ \\
\hline Neuro-inflammatory diseases & $\begin{array}{l}\text { Relatively common. } \\
\text { Explore specificity of putative } \\
\text { markers, especially glial. } \\
\text { May have 'mimic' potential e.g. } \\
\text { Primary Progressive MS } \\
\text { versus PLS }\end{array}$ & $\begin{array}{l}\text { Relevance of primary immune-based } \\
\text { conditions to ALS, including age, natural } \\
\text { history, treatments? }\end{array}$ \\
\hline
\end{tabular}

ALS: amyotrophic lateral sclerosis; FTD: frontotemporal dementia; MFMN: multifocal motor neuropathy with conduction block; MS: multiple sclerosis; PLS: primary lateral sclerosis.

especially for an age related condition like ALS. Some controls (e.g. spouses) will have the advantage of additional matching for environmental factors. Obtaining more than one control for every patient enrolled is recommended. It must be carefully considered whether to use blood relatives of patients as controls, even when studying apparently sporadic cases, as biomarkers might in theory be present in both the patient and an unaffected relative with an occult genetic predisposition. The establishment instead of specific repositories for samples from presymptomatic relatives of patients with known ALSassociated gene mutations is advocated, being a potentially powerful resource in understanding the earliest steps in pathogenesis and achieving the larger goal of disease prevention. This will require significant and sustained funding as well as a robust ethical and counseling framework.

\section{Longitudinal studies}

Multiple sampling over time in individual patients is the gold standard for assessing the usefulness of any biomarker as a prognostic indicator or marker of disease progression. It may not be an essential requirement to have a separate control group in longitudinal studies, since each patient can serve as their own control, with the change in value over time of a biomarker being the most relevant measure. The sampling interval must be a balance between the improved statistical robustness of multiple timepoints and the practicality of repeated sampling in patients who become increasingly disabled. Easily obtainable samples (e.g. blood, urine, saliva) might reasonably be sought every three months, whereas six-monthly or annual sampling is more realistic for more invasive procedures (e.g. cerebrospinal fluid (CSF), skin or muscle biopsy). At present, neurofilaments have been confirmed independently by a number of groups to be of prognostic and diagnostic value (e.g. 10-13). Therefore we recommend the quantification of the very stable neurofilament heavy chain to be included in longitudinal studies. Apart from diagnostic use this will allow comparison of studies, benchmarking of other candidates and emerging biomarkers and, if absent, indicate poor sample quality. Flexibility in timing of repeated samples and continued clinical record keeping in those who withdraw from the sampling aspects of the study are essential.

\section{Clinical data collection}

Biomarker studies are of limited or no use without reliable clinical characterization of patients and any controls, but exhaustive requirements for clinical data recording at sampling are generally a disincentive for busy physicians, and may discourage multicenter participation. Defining a 'minimal' datacet ic 
Table IV. Desirable minimum clinical dataset for ALS biomarker studies.

\begin{tabular}{|c|c|}
\hline Category & Clinical information \\
\hline $\begin{array}{l}\text { Generic (ALS } \\
\text { and controls) }\end{array}$ & $\begin{array}{l}\text { Age } \\
\text { Gender } \\
\text { Ethnicity } \\
\text { Drugs } \\
\text { Past medical history. } \\
\text { Family history of } \\
\quad \text { neurodegenerative disorders } \\
\quad \text { (back two generations) }\end{array}$ \\
\hline Phenotype (ALS) & $\begin{array}{l}\text { Diagnostic certainty (ALS by } \\
\text { revised El Escorial criteria } \\
\text { plus PLS or PMA), } \\
\text { confirmed each time-point. } \\
\text { Symptom onset (months/years) } \\
\text { Date of diagnosis } \\
\text { Site of onset (bulbar, upper/ } \\
\text { lower limb, respiratory) } \\
\text { Regional phenotypes (flail arm, } \\
\text { flail leg, progressive bulbar } \\
\text { palsy, upper versus lower } \\
\text { motor neuron-predominant } \\
\text { ALS, cognitive involvement } \\
\text { e.g. ALS-FTD) }\end{array}$ \\
\hline Evaluation (ALS) & $\begin{array}{l}\text { ALSFRS-R } \\
\text { Forced Vital Capacity } \\
\text { Upper/lower motor neuron } \\
\text { involvement } \\
\text { Body Mass Index } \\
\text { Cognitive assessment e.g. } \\
\text { Verbal Fluency Index }\end{array}$ \\
\hline Interventions (ALS) & $\begin{array}{l}\text { Gastrostomy } \\
\text { Non-invasive ventilation } \\
\text { Cough-assist device } \\
\text { Tracheostomy } \\
\text { Date of death (with } \\
\text { consideration of stratification } \\
\text { of patients according to time } \\
\text { of sampling in relation to } \\
\text { overall disease course) }\end{array}$ \\
\hline
\end{tabular}

ALS: amyotrophic lateral sclerosis; ALSFRS-R: revised ALS functional rating score;

FTD: frontotemporal dementia; PMA: progressive muscular atrophy; PLS: primary lateral sclerosis.

critical prior to a longitudinal study (see Table IV), where the anonymization procedures involved in multi-site studies generally preclude returning to individual subject clinical records at a later date.

\section{Choice of samples}

Although blood and CSF are currently the leading candidate sources for biomarkers across a range of neurological disorders, there are other sources in which relevant biomarkers are present. ALS biomarker research can be performed on urine samples, skin fibroblasts, muscle biopsy and post mortem tissue samples. Each of these starting materials offers particular advantages and disadvantages (see Table V).

\section{Standard operating procedures}

Standard operating procedures (SOPs) must be utilized for the collection, processing and storage of biofluid and tissue samples to be used in biomarker discovery and validation efforts. These SOPs must be established in each laboratory. Acceptable SOPs for CSF have recently been published (9). SOPs for blood collection and storage procedures are also established. Although questions remain regarding the value and practicality of standardizing fasting status and time of day for sample collection, such information, along with the processing 'timeto-freezer' should be recorded and linked to all samples in a database. All biofluids should be collected in polypropylene tubes with screw caps and stored at $-80^{\circ} \mathrm{C}$. While not all groups may be able to follow exactly identical collection, processing and storage protocols, it is crucial to at least record the volume and precise nature of the tissue sample. This provides opportunities to utilize search parameters within the biobank repository to optimize the samples retrieved and used in research studies. Any protocol exceptions should be recorded to permit research studies on the most homogeneous set of samples.

\section{Cerebrospinal fluid (CSF)}

A majority of ALS biomarker discovery research has been performed using CSF (for reviews see $(2-4))$. A frankly traumatic tap should be recorded, and good practice is to discard the first $2 \mathrm{ml}$ to minimize occult blood contamination (this can be sent for routine laboratory CSF parameters such as cell counts, total protein, albumin and lactate, with paired serum). The latter measurement can be used for internal and external quality control of biosamples (14). Larger volumes of CSF are most helpful and there is no risk in removing up to $20 \mathrm{ml}$. Less than $5 \mathrm{ml}$ can adversely affect quantitative measures due to gradient effects. The incidence of lifethreatening complications of lumbar puncture (in those without standard clinical contraindications) is negligible. Significant post-procedure headache is uncommon with the use of $22 \mathrm{G}$ or narrower needle gauge, and largely self-limiting within $72 \mathrm{~h}$ when it occurs. In one research study there was an incidence of only $2.5 \%$ in a cohort of over $1000 \mathrm{AD}$ study subjects (15), and so concern about complications should not be seen as an ethical barrier per se to the extraction of this valuable biomarker source.

The importance of rapidly centrifuging the CSF has been demonstrated in studies that identified rapid generation of proteomic and metabolomic artifacts $(16,17)$. The supernatant should be quickly aliquoted into polypropylene screw cap tubes and frozen within an hour. CSF should be centrifuged with a speed of up to $2000 \mathrm{~g}$ for at least $10 \mathrm{~min}$ (at $4^{\circ} \mathrm{C}$ if the resources are available), to pellet any cells and avoid their lysis with subsequent thawing. Prompt CSF handling is also important to minimize observed variations in $\mathrm{pH}$ (18). 
Table V. Strengths and weaknesses among biomarker sources.

\begin{tabular}{|c|c|c|c|c|c|c|}
\hline Characteristic & Blood $^{1}$ & CSF & Urine & Saliva & Skin & Muscle \\
\hline Proximity to CNS pathology & ++ & +++ & + & + & + & + \\
\hline Less molecular complexity & + & + & ++ & +++ & ++ & ++ \\
\hline Less invasive & ++ & + & +++ & +++ & + & + \\
\hline Practicality of sampling & +++ & ++ & +++ & ++ & + & + \\
\hline Ease of handling for storage & ++ & +2 & ++ & + & + & + \\
\hline Resistance to exogenous drug contamination & + & +++ & + & ++ & ++ & ++ \\
\hline Candidate molecules to date & ++ & +++ & + & + & + & + \\
\hline Potential for DNA/RNA analysis & +++ & + & + & ++ & +++ & +++ \\
\hline
\end{tabular}

+++ : highly significant; ++ : significant; + : low significance.

${ }^{1}$ Plasma versus serum needs to be specified; serum may have advantages for the stability of some proteins, e.g. immunoglobulins. EDTA sample will be needed for DNA or RNA studies.

${ }^{2}$ Bloody tap contamination potential and the particular need for rapid centrifugation.

\section{Blood}

The standardization of protocols for sampling, processing and storage must be tailored to the different blood components and bio-assays potentially relevant to the search for biomarkers in ALS. The blood collection tube type will determine if whole blood, serum or plasma has been processed for banking. Since hormonal circadian rhythm and diet are likely to influence the bioavailability of different compounds and of the gene expression in blood, a morning fasting blood sample may be ideal although probably not always possible during the regular follow-up of ALS patients. RNA analyses and metabolomics are thought to be most sensitive to time of day and the fasting state at collection. Proteomic analysis is less sensitive and DNA analysis least.

The impact of pre-analytical handling on mRNA gene expression has been evaluated with regard to the effects of delays to freezing and the buffers. Storage of whole blood at $-80^{\circ} \mathrm{C}$ is recommended, in either PAX-gene tubes or in RNAlater highly saline solution, the latter used in RNase-free microfuge tubes in which the RNAlater solution is three-fold the volume of blood. Red cells as a by-product of whole blood centrifugation during plasma extraction can be safely pooled into plastic tubes and stored at $-80^{\circ} \mathrm{C}$. This simple protocol allows lipid chemistry analysis in red cells. White cell separation may provide the best source for DNA/RNA extraction or serve as a test-bed in the search of peripheral inflammatory markers in ALS. Protocols for white cells separation can be implemented provided that the laboratory is equipped with the appropriate cryopreservation facilities (19).

\section{Urine}

Urine is an easily obtained source of potential biomarkers. Since urinary tract infections or an asymptomatic bacteriuria can negatively affect the results, the semi-quantitative determination of basic urine parameters including erythrocytes, leukocytes, protein, $\mathrm{pH}$, and nitrite is mandatory. Although a 24-h urine collection might be the ideal for quantitative analysis of urinary proteins, it is less practicable. Collection of morning urine (that includes biochemical substances from several hours' urine production) is recommended to overcome potential issues relating to diurnal variation. The second morning urine is recommended, as this reduces contamination of proteins from bacteria or bladder epithelial cells. The use of the creatinine ratio may compensate for 24-h urine collection. A correction factor of 1.2 was proposed due to a lower creatinine excretion in females.

\section{Muscle}

Muscle biopsies prepared for routine clinical histopathologic studies are flash-frozen in liquid nitrogen, prepared for electron microscopy, or fixed in formalin following procurement and stained according to rigorous SOPs. However, the histopathologic changes described in ALS are non-specific, occur late in the disease, and can often be measured less invasively with electrodiagnostic testing. Furthermore, it remains unknown whether muscle damage in ALS is due to unique biochemical pathways within the muscle or non-specific changes reflecting denervation (20). Thus, biomarker discovery on muscle tissue is likely to take the form of proteomic, metabolomic, gene expression, or other novel techniques.

\section{Post mortem tissues}

The provision of high-quality post mortem brain and spinal cord tissue from patients with amyotrophic lateral sclerosis (ALS) and other neurodegenerative diseases is vital to future research into the causes and treatment of these devastating illnesses. Such material is of particular importance in the elucidation of the molecular mechanisms involved in disease pathogenesis, and can verify the source of biomarker candidates identified in biofluids. Studies on post mortem CNS tissue are complementary to experiments in animal and in vitro models, and may provide the only confirmation of disease relevance. In vivo imaging techniques might also benefit from validation through post mortem examinotinn Hirh 
quality work using post mortem material can only be performed if the tissue is preserved soon after death and in such a way as to allow a wide range of techniques to be later applied to it, including mRNA extraction, protein studies, in situ hybridization, immunocytochemistry or laser capture microdissection; and fixed and/or paraffin embedded tissue blocks for histology and immunohistochemistry (2123). The primary objectives of CNS Tissue Banking in ALS should be to:

- Provide access to well documented brain and spinal cord tissues for research

- Provide optimal tissue for a wide range of scientific techniques and applications

- Increase the awareness of the importance of studying human brain tissue among patients and their caregivers (and crucially to reassure them that it will not delay funeral arrangements or affect the external body appearance)

- Optimize procedures for the recruitment of CNS tissue donors

- Minimize time from death to tissue procurement and freezing or fixation

- Pathologically confirm the disease status of the tissue

\section{Informed consent}

All studies involving human subjects require IRB/ REC approval, and there are specific considerations in relation to the storage and use of human tissue (although this does not usually include biofluids unless they have an appreciable cellular content), and separately for use of any genetic information. It is essential to consider obtaining specific consent for samples to be shared at a later date with local as well as international collaborators, including those focused on other neurodegenerative disorders. Recognition that such collaborations may be in the commercial sector is also important.

\section{Quality control}

This concept embraces the entire lifetime of a sample and putative biomarker and should include:

- pre-analytical tests such as stability in the discovery phase

- tests of accuracy of established analytical methods

- determination of reference values for validated tests, which may include participation in quality insurance schemes and strategies to audit cut-off values

- assurance that the accuracy of any assay should be better than the variation between groups

\section{Biomarker discovery platforms}

There are numerous technologies currently in use for ALS biomarker discovery and validation efforts.

\section{Proteomics}

To date, the most common methodological platform used for untargeted biomarker discovery efforts is proteomics. A schematic overview of quantitative proteomic approaches is shown in Figure 1. To date, the search for ALS biomarkers was mainly based on the surface enhanced laser desorption ionization (SELDI) technology (for review see (2)). While having the advantage that large numbers of individual samples from biofluids can be processed without prior pre-fractionation, SELDI as a pattern recognition tool usually does not directly reveal the identity of the differential protein peaks and huge efforts are often needed subsequently to achieve this task (24). Alternatively, the two-dimensional fluorescence difference gel electrophoresis (2D-DIGE) system, a protein profiling method that can be interfaced to mass spectrometric protein identification in a more direct way, has been used for differential proteome analysis of CSF in ALS (25).

2D gel-based approaches have limitations such as under-representation of certain protein classes, low sensitivity, and restricted multiplexing capabilities. To address these limitations, gel-free mass spectrometry (MS)-based quantification approaches of the shotgun type have been put forward, although often on the cost of information linked to protein integrity. These approaches usually rely on stable isotopes, either introduced by chemical tagging (e.g. iTRAQ) or metabolic labeling (e.g. SILAC) (Figure 2). Here, potential drawbacks include the variation of labeling efficiency in iTRAQ and the requirement of cultured cells for SILAC, preventing direct application to human material. In this regard, very recent spike-in versions of the SILAC approach (26) are promising at least for tissue samples, although some bias may be introduced through the reference proteome. With the advancements in liquid chromatography (LC) and MS, label-free quantification is currently emerging as a powerful technique with the potential to complement stable isotopebased approaches (see, for example (27) for an experimental comparison). Based on the softwareassisted comparison of peptide intensities within separate LC-MS/MS runs, this technique features high sensitivity and dynamic range, unlimited multiplexing capabilities, and low running costs, making it ideally suitable for biomarker discovery from biofluids. However, as the samples to be compared are not mixed at any time (as in stable isotope labeling workflows), accuracy of quantification is an issue and the inherently higher variability has to be compensated by technical replicates. An early form of label-free quantification has even been used for protein pattern classification and identification in CSF from ALS patients (28).

Biomarker discovery typically involves searching for low-abundance proteins and proteins of small molecular weight such as cytokines and 


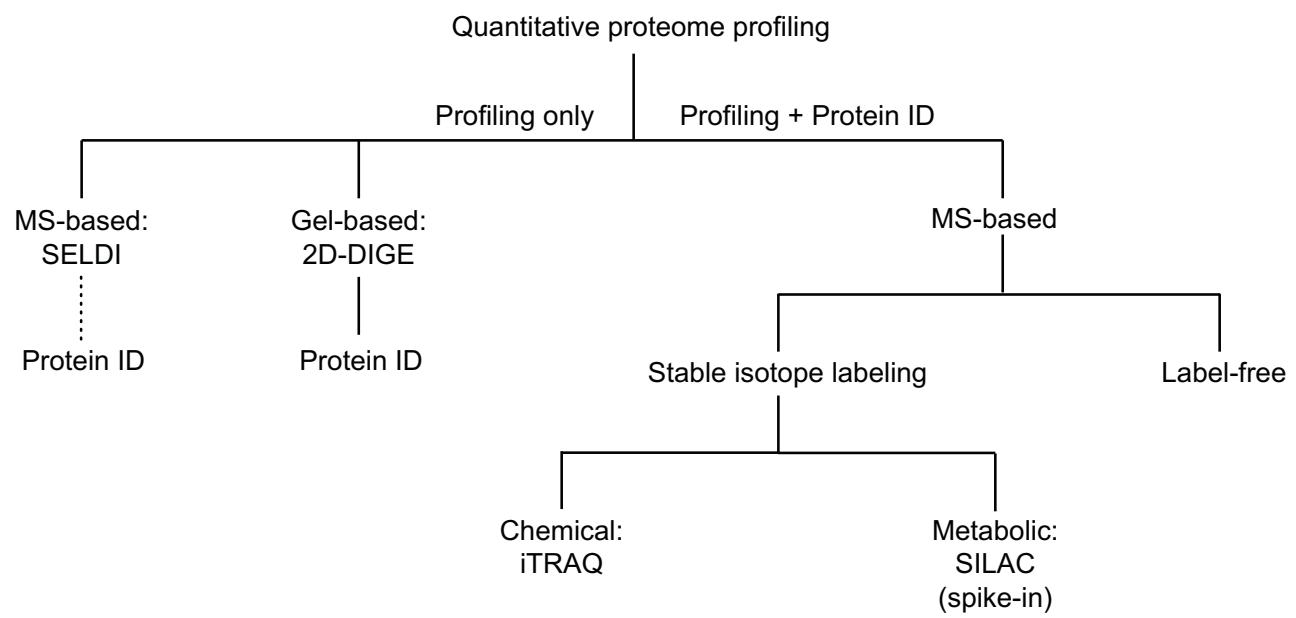

Figure 2. 'Quantification tree' showing the most common approaches for proteome profiling in discovery proteomics. SELDI and 2D-DIGE reveal only pattern information in the first instance, but 2D-DIGE (in contrast to SELDI) can be readily interfaced to mass spectrometric protein identification. MS-based approaches, which reveal both protein profile and identity, branch into stable isotope labeling-based and label-free techniques. Stable isotopes can be introduced by chemical tagging (e.g. iTRAQ) or by metabolic labeling (e.g. SILAC). SILAC can only be applied as a spike-in version to human material as it requires metabolic labeling of proteins in cell culture. For label-free quantification, peptide intensities are compared within separate LC-MS/MS runs.

SELDI: surface-enhanced laser desorption isonization; 2D-DIGE: two-dimensional fluorescence difference gel electrophoresis; MS: mass spectrometry; SILAC: stable isotope labeling with amino acids in cell culture; iTRAQ: isobaric tag for relative and absolute quantitation; LC: liquid chromatography.

trophic factors. Detection of these small and low abundant proteins - that can be detected with multiplex antibody based platforms - can provide critical data on disease states. A further advantage is, that this biomarker profile can't be overlooked $(29,30)$. A targeted proteomic approach is important to consider, given that the typical dynamic range of quantitative proteomic approaches (2-4 orders of magnitude) is far from being sufficient to cover the entire protein concentration range of CSF ( 8 orders of magnitude (2)) or plasma/serum (10 orders of magnitude). Such biofluids must be depleted of high-abundance proteins to shift the quantifiable portion of the proteome towards minor (potentially pathology-specific) components. Antibody based depletion of up to 20 major proteins and resin-based affinity removal of albumin and IgG are common pre-fractionation strategies for both serum (31) and CSF (32). More recently, an alternative strategy based on the use of a combinatorial hexapeptide ligand library has been introduced (33) and used for an in-depth exploration of the CSF proteome (34).

The targeted validation phase of biomarker discovery workflow has been expedited due to the more recent transfer of the well-established selected reaction monitoring technology (SRM, also known as multiple reaction monitoring - MRM) from the small molecule field into proteomics (35). This is an easy-to-operate mass spectrometric method for the accurate quantitation of (pre-defined) proteins from complex mixtures, with the potential to overcome antibody-based assays bottlenecks of biomarker validation (which typically involve antibody-based assays). For absolute quantitation, SRM assays require stable isotope-labeled standards of the targeted proteotypic peptides, a high demand that hampered broad applicability of SRM-MS in routine clinical proteomics to date. This is expected to change with the success of international initiatives like the human Proteome Detection and Quantitation Project (hPDQ) so that in future SRM-MS may bridge the gap between discovery proteomics and clinical assay development.

The proteomic and perhaps any of the biomarker analyses are unlikely to identify a single protein that will provide diagnostic and therapeutic insights but rather a biomarker panel or 'fingerprint' is more likely. Therefore, data analysis is as critical as the platform chosen to provide the biomarker. For example, multifactorial analysis of analyte expression and crossvalidation in a 'leave one out' strategy for determining how well a protein panel (fingerprint) is associated with disease classification, should be considered as the biomarker strategies go forward $(29,30)$.

\section{$R N A$ and DNA analysis}

Biomarker discovery in the central nervous system using the available micro-array platforms for tissue RNA-profiling is limited by the cell based heterogeneity and complexity. To circumvent this problem and obtain cell-specific markers of disease initiation and progression, laser-capture microdissection has become a viable route to explore the contribution to the pathology of different cellular entities (36). The discovery of the role of particularly vulnerable portions of RNA like the microRNAs, non-coding RNAs that post-transcriptionally regulate gene expression and may contribute to human diseases including ALS (37), has accentuated the debate on the reliability of the commonly used techniques of RNA 
extraction and storage and their effect on RNA integrity.

Similar to other human disorders, ALS may arise from the acquisition of somatic, genetic and epigenetic alterations leading to changes in gene sequence, structure, copy number and expression. Therefore, the most appropriate investigative tools must allow serial analysis of gene expression in parallel with high-throughput DNA sequencing to define point mutations, rearrangements and copy-number changes. As systems biology evolves into the definition of new types of biological macromolecules, a comprehensive analysis of transcriptomes will have to include the characterization of microRNAs and of protein-DNA interactions. miRNA and mRNA in exosomes are probably more stable and therefore more suitable for biomarker studies.

\section{Metabolomics}

Metabolomics is a complementary technique to genomics, transcriptomics and proteomics that aims to study the metabolome in a holistic way. The first global metabolomic studies in relation to ALS reported reduced levels of metabolites and point towards a general depletion of metabolites in blood (38) and CSF (39). For metabolomic analysis even stricter preanalytic precautions have to be undertaken and were set up by the Metabolomic Standards initiative (40).

\section{Lipidomics}

The role of lipids in cells and tissue physiology is demonstrated by a large number of studies confirming the disruption of lipid metabolic enzymes and pathways in the development of several human conditions, including neurodegenerative disorders. While strong evidence linking a disrupted lipid homeostasis to an early alteration of the metabolic state is emerging in ALS, deregulated lipid metabolism has been long known to be of particular importance in brain injuries and in the development of other neurodegenerative conditions. The introduction of tandem mass spectrometry (MS) and of liquid chromatography for the resolution of lipid chemistry has revolutionized this area of investigation, creating the ground for the large-scale profiling of a vast array of lipid classes and the computational demand for handling the large amount of data that these techniques generate (41).

One of the most comprehensive repositories of information surrounding lipid classifications, metabolic pathways, involvement in disease processes is contained in the Nature Lipidomics Gateway (http:// www.lipidmaps.org). In this web-based resource, practical considerations are discussed with respect to methods of sampling, storage, and lipid extraction of red blood cells. An overview of analysis tools for lipid determination, of analytical standards to obtain MS spectra and of the experimental parameters used for acquisition of MS/MS spectra is also available.

A caveat in the reliable use of lipidomics to dissect novel disease biomarkers is the largely untested effect that diet may have in the lipid profile of the target biological fluids or tissues. Diet can cause short- and long-term effects, introducing 'confounding noises' that could interfere with the disease related changes of specific lipid compounds or mediators (42).

\section{Conclusions}

Biomarker discovery and biobanking are now at the forefront of the global research effort in ALS. Standardized procedures are an essential part of fostering the large collaborative effort necessary for a rare disorder such as ALS. Comprehensive clinical information collated by neurologists with ALS expertise is equally vital. Biomarker discovery and validation is costly. As well as intellectual cooperation among scientists, and the continued encouragement of patients to take part in such studies, leadership and vision are needed to put forward the imperative that this is a vital investment in tackling the imminent global increase in the prevalence of neurodegenerative disorders.

\section{Acknowlegements}

This work was supported by World Federation of Neurology. The name Volcano Group was chosen because the first meeting of the group had to be postponed as several group members were stranded somewhere in the world because of the eruption of the Eyjafjallajökull in Iceland in April 2010.

We also acknowledge the comment of Mamede de Carvalho.

Declaration of interest: The authors report no conflicts of interest. The authors alone are responsible for the content and writing of the paper.

\section{References}

1. Poser CM, Johnson M, Bunch LD. Serum Amino Acid Studies in Amyotrophic Lateral Sclerosis I. Results of Arginine Tolerance Tests. Arch Neurol. 1965;12:604-9.

2. Bowser R, Lacomis D. Applying proteomics to the diagnosis and treatment of ALS and related diseases. Muscle Nerve. 2009;40:753-62.

3. Ryberg H, Bowser R. Protein biomarkers for amyotrophic lateral sclerosis. Expert Rev Proteomics. 2008;5:249-62.

4. Turner MR, Kiernan MC, Leigh PN, Talbot K. Biomarkers in amyotrophic lateral sclerosis. Lancet Neurol. 2009;8:94-109.

5. Kiernan MC, Vucic S, Cheah BC, Turner MR, Eisen A, Hardiman O, et al. Amyotrophic lateral sclerosis. Lancet. 2011 (Early Online Publication, February 7 2011).

6. Poste G. Bring on the biomarkers. Nature. 2011;469: 156-7.

7. Turner MR, Grosskreutz J, Kassubek J, Abrahams S, Agosta F, Benatar M, et al. Towards a neuroimaging biomarker in amyotrophic lateral sclerosis. Lancet Neurol. 2011;10:400-3. 
8. Sherman A, Bowser R, Grasso D, Power B, Milligan C, Jaffa M, et al. Proposed BioRepository platform solution for the ALS research community. Amyotroph Lateral Scler. 2011;12:11-6.

9. Teunissen CE, Petzold A, Bennett JL, Berven FS, Brundin L, Comabella M, et al. A consensus protocol for the standardization of cerebrospinal fluid collection and biobanking. Neurology. 2009;73:1914-22.

10. Brettschneider J, Petzold A, Sussmuth SD, Ludolph AC, Tumani H. Axonal damage markers in cerebrospinal fluid are increased in ALS. Neurology. 2006;66:852-6.

11. Ganesalingam J, An J, Shaw CE, Shaw G, Lacomis D, Bowser R. Combination of neurofilament heavy chain and complement C3 as CSF biomarkers for ALS. J Neurochem. 2011;117:528-37.

12. Petzold A. Neurofilament phosphoforms: surrogate markers for axonal injury, degeneration and loss. J Neurol Sci. 2005;233:183-98.

13. Boylan K, Yang C, Crook J, Overstreet K, Heckman M, Wang Y, et al. Immunoreactivity of the phosphorylated axonal neurofilament $\mathrm{H}$ subunit ( $\mathrm{pNF}-\mathrm{H}$ ) in blood of ALS model rodents and ALS patients: evaluation of blood pNF-H as a potential ALS biomarker. J Neurochem. 2009;111:1182-91.

14. Jesse S, Brettschneider J, Sussmuth SD, Landwehrmeyer BG, von Arnim CA, Ludolph AC, et al. Summary of cerebrospinal fluid routine parameters in neurodegenerative diseases. J Neurol. 2011;258:1034-41.

15. Zetterberg H, Tullhog $\mathrm{K}$, Hansson O, Minthon L, Londos E, Blennow K. Low incidence of post-lumbar puncture headache in 1089 consecutive memory clinic patients. Eur Neurol. 2010;63:326-30.

16. Ranganathan S, Polshyna A, Nicholl G, Lyons-Weiler J, Bowser R. Assessment of protein stability in cerebrospinal fluid using surface-enhanced laser desorption/ionization time-of-flight mass spectrometry protein profiling. Clin Proteomics. 2006;2:91-101.

17. Rosenling T, Slim CL, Christin C, Coulier L, Shi S, Stoop $\mathrm{MP}$, et al. The effect of preanalytical factors on stability of the proteome and selected metabolites in cerebrospinal fluid (CSF). J Proteome Res. 2009;8:5511-22.

18. Wuolikainen A, Hedenstrom M, Moritz T, Marklund SL, Antti H, Andersen PM. Optimization of procedures for collecting and storing of CSF for studying the metabolome in ALS. Amyotroph Lateral Scler. 2009;10:229-36.

19. Elliott P, Peakman TC. The UK Biobank sample handling and storage protocol for the collection, processing and archiving of human blood and urine. Int J Epidemiol. 2008;37:234-44.

20. Wong M, Martin LJ. Skeletal muscle-restricted expression of human SOD1 causes motor neuron degeneration in transgenic mice. Hum Mol Genet. 2010;19:2284-302.

21. Mirra SS, Heyman A, McKeel D, Sumi SM, Crain BJ, Brownlee LM, et al. The Consortium to Establish a Registry for Alzheimer's Disease (CERAD). Part II. Standardization of the neuropathologic assessment of Alzheimer's disease. Neurology. 1991;41:479-86.

-22. Ferrer I, Santpere G, Arzberger T, Bell J, Blanco R, Boluda $S$, et al. Brain protein preservation largely depends on the post mortem storage temperature: implications for study of proteins in human neurologic diseases and management of brain banks: a BrainNet Europe Study. J Neuropathol Exp Neurol. 2007;66:35-46.

23. Durrenberger PF, Fernando S, Kashefi SN, Ferrer I, Hauw JJ, Seilhean D, et al. Effects of ante mortem and post mortem variables on human brain mRNA quality: a BrainNet Europe study. J Neuropathol Exp Neurol. 2010;69:70-81.

24. Steinacker P, Rist W, Swiatek-de-Lange M, Lehnert S, Jesse S, Pabst A, et al. Ubiquitin as potential cerebrospinal fluid marker of Creutzfeldt-Jakob disease. Proteomics. 2010;10: 81-9.

25. Tumani H, Lehmensiek V, Lehnert S, Otto M, Brettschneider J. 2D DIGE of the cerebrospinal fluid proteome in neurological diseases. Expert Rev Proteomics. 2010;7:29-38.

26. Geiger T, Wisniewski JR, Cox J, Zanivan S, Kruger M, Ishihama Y, et al. Use of stable isotope labeling by amino acids in cell culture as a spike-in standard in quantitative proteomics. Nat Protoc. 2011;6:147-57.

27. Patel VJ, Thalassinos K, Slade SE, Connolly JB, Crombie A, Murrell JC, et al. A comparison of labeling and label-free mass spectrometry-based proteomics approaches. J Proteome Res. 2009;8:3752-9.

28. Ramstrom M, Ivonin I, Johansson A, Askmark H, Markides $\mathrm{KE}$, Zubarev R, et al. Cerebrospinal fluid protein patterns in neurodegenerative disease revealed by liquid chromatography-Fourier transform ion cyclotron resonance mass spectrometry. Proteomics. 2004;4:4010-8.

29. Mitchell RM, Freeman WM, Randazzo WT, Stephens HE, Beard JL, Simmons Z, et al. A CSF biomarker panel for identification of patients with amyotrophic lateral sclerosis. Neurology. 2009;72:14-9.

30. Mitchell RM, Simmons Z, Beard JL, Stephens HE, Connor JR. Plasma biomarkers associated with ALS and their relationship to iron homeostasis. Muscle Nerve. 2010;42:95-103.

31. Levin Y, Wang L, Schwarz E, Koethe D, Leweke FM, Bahn S. Global proteomic profiling reveals altered proteomic signature in schizophrenia serum. Mol Psychiatry. 2009;15:1088-100.

32. Brechlin P, Jahn O, Steinacker P, Cepek L, Kratzin H, Lehnert $\mathrm{S}$, et al. Cerebrospinal fluid-optimized twodimensional difference gel electrophoresis (2-D DIGE) facilitates the differential diagnosis of Creutzfeldt-Jakob disease. Proteomics. 2008;8:4357-66.

33. Guerrier L, Righetti PG, Boschetti E. Reduction of dynamic protein concentration range of biological extracts for the discovery of low-abundance proteins by means of hexapeptide ligand library. Nat Protoc. 2008;3:883-90.

34. Mouton-Barbosa E, Roux-Dalvai F, Bouyssie D, Berger F, Schmidt E, Righetti PG, et al. In-depth exploration of cerebrospinal fluid by combining peptide ligand library treatment and label-free protein quantification. Mol Cell Proteomics. 2010;9:1006-21.

35. Lange V, Picotti P, Domon B, Aebersold R. Selected reaction monitoring for quantitative proteomics: a tutorial. Mol Syst Biol. 2008;4:222.

-36. Espina V, Heiby M, Pierobon M, Liotta LA. Laser capture microdissection technology. Expert Rev Mol Diagn. 2007;7:647-57.

-37. Williams AH, Valdez G, Moresi V, Qi X, McAnally J, Elliott JL, et al. MicroRNA-206 delays ALS progression and promotes regeneration of neuromuscular synapses in mice. Science. 2009;326:1549-54.

38. Rozen S, Cudkowicz ME, Bogdanov M, Matson WR, Kristal BS, Beecher C, et al. Metabolomic analysis and signatures in motor neuron disease. Metabolomics. 2005;1:101-8.

39. Wuolikainen A, Moritz $T$, Marklund SL, Antti $H$, Andersen PM. Disease-related changes in the cerebrospinal fluid metabolome in amyotrophic lateral sclerosis detected by GC/TOFMS. PLoS One. 2011;6:17947.

40. Sansone SA, Fan T, Goodacre R, Griffin JL, Hardy NW, Kaddurah-Daouk R, et al. The metabolomics standards initiative. Nat Biotechnol. 2007;25:846-8.

41. Quinn PJ, Rainteau D, Wolf C. Lipidomics of the red cell in diagnosis of human disorders. Methods Mol Biol. 2009;579:127-59.

42. Hodson L, Skeaff CM, Fielding BA. Fatty acid composition of adipose tissue and blood in humans and its use as a biomarker of dietary intake. Prog Lipid Res. 2008;47:348-80. 